\title{
Influence of the Degreaser Type on the Bonded Joint Strength
}

Milan Brožek

Department of Material Science and Manufacturing Technology, Faculty of Engineering, Czech University of Life Sciences Prague, Kamýcká 129, 16521 Praha 6 - Suchdol, Czech Republic, E-mail: brozek@tf.czu.cz

In the report the results of bonded joints laboratory tests are published. The determination of the tensile lap-shear strength of rigid-to-rigid bonded assemblies according to the standard CSN EN 1465 (66 8510) was the aim of the carried out tests. The samples were made from steel sheets. For bonding of test samples four different types of adhesives were used (two super glue adhesives, one epoxy adhesive and one contact adhesive). Ahead of bonding the surfaces of all samples were blasted using corundum grit and degreased. For degreasing four various types of five different degreasers were used, namely perchlorethylene, acetone, methanol, technical gasoline and toluene. Then the samples were dried using warm air. For comparison the blasted samples without degreasing were bonded, too. At the tested samples bonding the consumption of adhesive was determined. From the adhesive consumption for one bonded joint and from the adhesive price the costs of one sample bonding were calculated. After curing the bonded assemblies were loaded using the universal test machine LabTest 5.50 ST up to their rupture. The rupture force was written down. From the values of the rupture force and the bonded surface size the bonded joint strength was calculated. By the carried out tests evaluation the influence of different degreaser types was determined. Also for all used adhesives the price of one bonded joint was calculated.

Keywords: adhesive bonding, degreasing, technological properties of adhesives, testing of bonded joints

\section{References}

[1] BROCKMANN, W. et al. (2009). Adhesive bonding: materials, applications and technology. Weinheim, Wiley$\mathrm{VCH}$.

[2] COGNARD, P. (2006). Adhesives and sealants: general knowledge, application techniques, new curing techniques. Oxford, Elsevier.

[3] KOVAČIČ, Ĺ. (1984). Lepenie kovov a plastov (Adhesive bonding of metals and plastics). 2nd Ed., Bratislava, Alfa.

[4] OSTEN, M. (1996). Práce s lepidly a tmely (Work with adhesives and cements). Praha, Grada Publishing.

[5] PETERKA, J. (1980). Lepení konstrukčních materiáli̊ ve strojírenství (Bonding of construction materials in engineering). Praha, SNTL.

[6] BERANEK, L., KYNCL, J., MIKES, P. (2014). Analysis of HVOF Coating on Molds Used for Refractory Fireclay Shapes. Manufacturing Technology. 14. 3. 267-271.

[7] BROŽEK, M. (2011). Layer number influence on weld deposit chemical composition. In.: Engineering for Rural Development. 3.-6. May. Latvia University of Agriculture. 393-397.

[8] BROŽEK, M. (2012). Wear resistance of multi-layer overlays. In.: Engineering for Rural Development. 24-25. May. Latvia University of Agriculture. 210-215.

[9] BROŽEK, M., NOVÁKOVÁ, A., MIKUŠ, R. (2010). Study of wear resistance of hard facings using welding powders on the NiCrBSi basis. In.: Trends in Agricultural Engineering. 7.-10. September. Czech University of Life Sciences Prague. 115-118.

[10] BROŽEK, M. (2014). Working variables optimization of resistance spot welding. Manufacturing Technology. 14. 4. 522-527.

[11] BROŽEK, M. (2013). Soldering sheets using soft solders. Acta Universitatis Agriculturae et Silviculturae Mendelianae Brunensis, 61. 6. 1597-1604.

[12] BROŽEK, M. (2013). Optimization of adhesive layer thickness at metal bonding using quick-setting adhesives. Manufacturing Technology. 13. 4. 419-423.

[13] BROŽEK, M. (2003). Studium faktorů ovlivňujících pevnost lepených spojů (Study of factors influencing the bonded joint strength). Strojárstvo/Strojírenství. 7. 7/8. 54-55. 
[14] BROŽEK, M. (2003). Vliv tloušt'ky vrstvy lepidla na pevnost lepeného spoje (Influence of adhesive layer thickness on bonded joint strength). MM Prümyslové spektrum. 1/2. 72.

[15] BROŽEK, M. (2003). Vliv doby vytvrzování lepidla na pevnost lepených spojů (Influence of adhesive curing time on bonded joint strength). MM Prümyslové spektrum. 7/8. 67.

[16] BROŽEK, M. (2013). Technical-economical evaluation of plywood bonding. In.: Trends in Agricultural Engineering. 3.-6. September. Czech University of Life Sciences Prague. 100-105.

[17] BROŽEK, M., MULLER, M. (2004). Mechanické vlastnosti spojů lepených sekundovými lepidly. Strojírenská technologie. IX. 1. 9-15.

[18] KAŠPAR, Z., BROŽEK, M. (2005). Vliv drsnosti povrchu hliníku na pevnost lepených spojů. Strojírenská technologie. X. 3. 5-10.

[19] MULLER, M., BROŽEK, M. (2005). Technologie lepení - vliv expirační doby na pevnost lepených spojů. Strojírenská technologie. X. 3. 10-16.

[20] MULLER, M., BROŽEK, M., VALÍČEK, J. (2009). Interakce vlivu integrity lepeného povrchu a tloušt'ky vrstvy lepidla na proces lepení. Strojírenská technologie. XIV. 3. 18-25.

[21] NOVÁKOVÁ, A., BROŽEK, M. (2009). Bonding of non-metallic materials using thermoplastic adhesives. In.: Engineering for Rural Development. 28.-29. May. Latvia University of Agriculture. 261-264.

[22] JOHNSON, K. (1973). Dry cleaning and degreasing chemicals and processes. Park Ridge, Noyes Data.

[23] SOUKUP, M., RUML, V. (1978). Odmašt’ování v průmyslu a zemědělství (Degreasing in industry and agriculture). Praha, SNTL.

[24] EBNESAJJAD, S. (2011). Handbook of adhesives and surface preparation: technology, applications and manufacturing. Oxford, William Andrew.

[25] CAGLE, Ch.V. (1973). Handbook of adhesive bonding. New York, Mac-Graw-Hill.

[26] EBNESAJJAD, S. (2008). Adhesives technology handbook. 2nd Ed., Norwich, William Andrew.

[27] EPSTEIN, G. (1954). Adhesive bonding of metals. New York, Reinhold.

[28] ANDERSON, G.P., BENNETT, S.J., DeVRIES, K.L. (1977). Analysis and testing of adhesive bonds. New York, Academic Press.

[29] HABENICHT, G. (2009). Applied adhesive bonding, a practical guide for flawless results. Weinheim, Wiley$\mathrm{VCH}$.

[30] KŘÍŽ, R. (1994). Strojírenská přiručka (Engineering handbook). Vol. 5. Praha, Scientia.

[31] LOCTITE (1988). Der Loctite. München, Loctite.

[32] MELEZÍNEK, O. (1961). Lepení kovů ve strojírenství (Adhesive bonding of metals in engineering). Praha, SNTL.

[33] PIZZI, A., MITTAL, K.L. (2003). Handbook of adhesive technology. Dekker, New York.

[34] SADEK, M. (1987). Industrial application of adhesive bonding. London, Elsevier Applied Sciency.

[35] ČSN EN 1465 (2009). Lepidla - Stanovení pevnosti ve smyku při tahovém namáhání přeplátovaných lepených soustav (Adhesives - Determination of tensile lap-shear strength of bonded assemblies). ČNI. 